\title{
Micro-PIXE with 3.5 MeV protons for the study of low copper concentrations in atherosclerotic artery
}

\author{
M.D. Ynsa ${ }^{\text {a,b, }}$, J. Ripoll-Sau ${ }^{\text {a }}$, T. Osipowicz ${ }^{c}$, M.B.H. Breese ${ }^{c, d}$, Minqin Ren $^{c}$ \\ ${ }^{\text {a }}$ Departamento de Física Aplicada and Centro de Microanálisis de Materiales (CMAM), Universidad Autónoma de Madrid, Madrid, Spain \\ ${ }^{\mathrm{b}}$ Instituto Nicolás Cabrera, Universidad Autónoma de Madrid, Madrid, Spain \\ ${ }^{\mathrm{c}}$ Centre for Ion Beam Applications, Department of Physics, National University of Singapore, Singapore, 117551, Singapore \\ ${ }^{\mathrm{d}}$ Singapore Synchrotron Light Source, National University of Singapore, 5 Research Link, 117603, Singapore
}

\section{A R T I C L E I N F O}

\section{Keywords:}

Atherosclerosis

Zinc supplement

Lesion area

Copper

Iron

Zinc

Nuclear microscopy

Free radicals

\begin{abstract}
A B S T R A C T
The onset and progression of many degenerative diseases including atherosclerosis, have been shown to directly link to the presence/absence of certain metal ions. Consequently, the detection of these ions in tissues may improve the understanding of the driving pathophysiology. The $\mathrm{Cu}$ content during atherosclerosis development has not been studied due to its low concentration involved. In this work, the Cu level in atherosclerotic rabbit tissue is determined using PIXE with a $3.5 \mathrm{MeV}$ proton beam. The arteries of three animal groups fed with different diets were studied: group 1, rabbits on normal standard diet, group 2, on High Fat Diet (HFD) and group 3, on HFD + Zinc diet. Zinc supplement has been proven to inhibit the beginning of atherosclerotic lesion. The result of this study shows that the $\mathrm{Cu}$ levels in all the atherosclerotic lesions were lower than that in the arterial walls of the samples in HFD group.
\end{abstract}

\section{Introduction}

Atherosclerosis is one of the most common chronic coronary diseases. The innermost layer of the artery wall is affected by focal deposits of atherosclerotic material, mainly cholesterol esters, and fibrous material such as collagen. The oxidative processes are decisive in the development of atherosclerosis, through oxidation of low-density lipoproteins (LDL) and the formation of reactive oxygen species (ROS) [1].

The role of transition metals e.g. iron (Fe), zinc ( $\mathrm{Zn})$ and copper $(\mathrm{Cu})$ in the oxidation processes is indisputable [2]. The Fe and $\mathrm{Zn}$ levels measured in atherosclerotic plaques support the concept that $\mathrm{Zn}$ could have a protective effect due to the decrease of Fe concentration observed in the lesions of animals on Zinc-rich diet, probably inhibiting free radical activities catalyzed by access Fe after the animal models were put on such a diet for 8 weeks [3]. Cu is essential for the proper function of the enzymes which catalyze the oxidative reactions of LDL and highdensity lipoprotein (HDL). HDL is considered to be more sensitive to oxidation by $\mathrm{Cu}$ than LDL $[2,4]$. The relationship between the $\mathrm{Cu}$ content and the cardiovascular diseases is indisputable, and both the increase and the decrease of this element can be harmful. Previous studies have found an association between different cardiovascular problems and a low- $\mathrm{Cu}$ diet [5]. Whereas the $\mathrm{Cu}$ concentrations increased in advanced human carotid atherosclerotic lesions with regard to healthy arteries [6] and in the serum of the patients with severity atherosclerosis [7]. Consequently, the determination of $\mathrm{Cu}$ content in the atherosclerotic plaque and its adjacent arterial wall could therefore shed much light on the role of metals in the atherosclerosis.

PIXE (Particle Induced X-ray Emission) is an excellent analytical technique for the detection and quantification of low concentration metal in biological tissues, because it allows to map trace element concentrations at the ppm level. The process is similar to EDX (Energydispersive X-ray spectroscopy) where an electron beam excites the sample atoms, but PIXE uses protons to eject inner shell electrons. This leads to an orders of magnitude reduction in the bremsstrahlung background under the characteristic X-ray lines compared to EDX and consequently to a much better elemental sensitivity. PIXE trace sensitivity depends on the ion type and the energy of the analyzing beam [8], as well as the matrix composition of the analyzed sample. Generally, the best sensitivities are obtained with protons on samples with low-Z matrices ( $, \mathrm{H}, \mathrm{N}, \mathrm{O}$ ) such as biological samples which exhibit very low limits of detection. Currently, due to radiation protection regulations and the technical features of the available accelerators, the proton energies most often used for PIXE analysis are in the range of 2 to $3 \mathrm{MeV}$. The X-ray production cross section for inner ionizations increases with

\footnotetext{
* Corresponding author.

E-mail address: m.ynsa@uam.es (M.D. Ynsa).
} 
the proton energy in this range, therefore in order to optimize the sensitivity of particular elements, it is important to carefully choose the bombarding energy of protons.

PIXE with a proton beam of $2.1 \mathrm{MeV}$ has been previously employed to measure the trace metal contents in atherosclerotic tissue on a larger scale including lesion and arterial wall [3]. The results of this work led to a subsequent research project involving human trials on Zinc supplement at the National University Hospital in Singapore. However, the relatively low $\mathrm{Cu} \mathrm{X}$-ray production cross section for a proton energy of $2.1 \mathrm{MeV}$ was not sufficient to quantify the minute amount of $\mathrm{Cu}$ in the areas of interest of the blood vessel samples. To resolve this constraint, PIXE with a proton energy of more than $3 \mathrm{MeV}$ was exploited and this is technically possible at the CMAM facilities $[9,10]$.

In the present study, $3.5 \mathrm{MeV}$ protons were used to determine the Fe, $\mathrm{Cu}$ and $\mathrm{Zn}$ concentrations in artery samples of rabbits fed with a high cholesterol diet and supplemented with Zinc. The aim of this work is to assess diet effect on $\mathrm{Fe}, \mathrm{Cu}$ and $\mathrm{Zn}$ concentrations in artery sections. The determined $\mathrm{Cu}$ concentrations have supplied new information whereas $\mathrm{Fe}$ and $\mathrm{Zn}$ values have been compared with our previous results. The results of the current study where $\mathrm{Cu}$ has been determined strengthen and complement the earlier conclusions from our previous analyses with 2.1 MeV protons [3].

\section{Material and methods}

New Zealand white male rabbits, weighing on average $2.5 \mathrm{~kg}$, were obtained from the Laboratory Animal Centre (Sembawang, Singapore) and divided randomly into three groups. The first group was fed a normal standard diet; the second group was fed with high fat diet (HFD) SF00-221 (modified guinea pig and rabbit $+1 \%$ cholesterol); the third group was fed a Zinc-supplemented diet SF03-017 (modified guinea pig and rabbit $+1 \%$ cholesterol $+1000 \mathrm{ppm}(1 \mathrm{~g} / \mathrm{kg})$ zinc as zinc carbonate). These diets were purchased from Glen Forrest Stockfeeders, Western Australia. The food intake and animal body weights were monitored, and all rabbits gained weight during the experimental period of 8 weeks. No significant difference in the food intake was observed between the animals fed on the HFD and the group fed on high-fat diet plus Zinc [3].

During the 8-week feeding period, hemoglobin and blood $\mathrm{Zn}$ levels were monitored every 2 weeks, and at the end of the 8-week animal feeding period a range of blood parameters was measured. Further details of the sample preparation can be found [3].

The aorta arch was removed and cut into 3 segments A, B and C of about $5 \mathrm{~mm}$ in length for each segment. A schematic drawing is shown in reference [11]. Segments were flushed with deionized water to remove residual blood from the inner artery wall, and flash frozen in liquid nitrogen. Sections of the aortic arch were taken using a Leica CM3050S cryostat. For this work, sections of the A segment, closest to the heart, and the B segment, the mid segment, was studied. The sections for nuclear microscopy measurements were picked up on pioloform-coated nuclear microscopy target holders, for elemental mapping and concentration analysis. Serial sections were picked up on gelatin-coated slides and stained with H\&E (Hematoxylin and Eosin). The lesion area measurements were carried out on the stained sections using the Carl Zeiss Axiophot 2 image analyzer utilizing the KS400 (version 3.18) analysis software. As in former studies [3,11], the artery segment which exhibited the largest lesion area according to the area analysis was chosen for nuclear microscopy analysis. Serial sections of aortic arch were taken, one set for H\&E staining, while serial unstained aortic artery sections were used for elemental analysis.

The samples for the current study were carefully chosen to represent the different groups of animals.

The microbeam analyses were performed with $3.5 \mathrm{MeV}$ protons focused to a $3 \times 3 \mu \mathrm{m}^{2}$ beam with a proton current of about $500 \mathrm{pA}$, using the CMAM internal microbeam line [9]. Different regions of interest (ROI) with a size of $120 \times 120 \mu \mathrm{m}^{2}$ of the artery sections were selected for quantitative analysis and analyses using PIXE and backscattered protons (PBS) were carried out simultaneously. PBS provides information on matrix (major elements of the sample) composition, sample thickness and incident charge, and is used in conjunction with PIXE to quantify the trace element concentration results. PIXE spectra were collected using a low energy Ge detector placed at $135^{\circ}$ with and active area of $100 \mathrm{~mm}^{2}$, a $32 \mu \mathrm{m}$ Be window and a $120 \mu \mathrm{m}$ kapton filter to attenuate X-rays from light elements, which are abundant in biological tissue. Backscattered protons were detected using a surface barrier detector with an active area of $300 \mathrm{~mm}^{2}$ at a backscattering angle of $135^{\circ}$ to the beam, in Cornell geometry. All signals were recorded together with the beam position information using the MPSYS data acquisition system [12].

Data analysis was carried out using GUPIX-WIN code [13,14] for PIXE spectra and SIMNRA [15] for PBS spectra.

\section{Results and discussion}

\subsection{Cross section and spectrum background study}

In order to evaluate the enhancement of PIXE sensitivity with the higher proton energy of $3.5 \mathrm{MeV}$ instead of $2.1 \mathrm{MeV}$, the X-ray production cross sections of $\mathrm{Ca}, \mathrm{Fe}, \mathrm{Cu}$ and $\mathrm{Zn}$ for protons $2.1 \mathrm{MeV}$ and 3.5 $\mathrm{MeV}$ were calculated using computer code GUPIX. Table 1 shows the cross sections for the two energies (the uncertainty of the values is about $3-5 \%$ ) and the ratio of the values between both energies. The increase of the cross sections with energy becomes more acute as the atomic number increases. For a $3.5 \mathrm{MeV}$ beam, the $\mathrm{Cu}$ the cross section is a factor of 2.65 higher than that of a $2.1 \mathrm{MeV}$ beam, and for $\mathrm{Zn}$ a factor of 2.76 is found. However, the increase in atomic bremsstrahlung processes with proton energy also leads to an increase of the background in the PIXE spectra under the $\mathrm{K}$ peaks. This worsens the signal to noise ratio, and therefore the sensitivity of the technique [16].

PIXE spectra of the same region of an artery section with $2.1 \mathrm{MeV}$ and $3.5 \mathrm{MeV}$ protons, both are charged normalized, are compared in (Fig. 1). Clearly, the background has a maximum around the Ca-K X-ray energies, and is significantly higher at $3.5 \mathrm{MeV}$ than at $2.1 \mathrm{MeV}$. Nevertheless, while both the backgrounds under and the intensity of the $\mathrm{Cu}-\mathrm{K}_{\alpha}$ peak are insignificant when protons of $2.1 \mathrm{MeV}$ energy are used, the $3.5 \mathrm{MeV}$ spectrum clearly shows a $\mathrm{Cu}-\mathrm{K}_{\alpha}$ peak on top of the continuous background. This allows the quantification of $\mathrm{Cu}$, albeit with a relatively large uncertainty (our criteria to consider $\mathrm{Cu}$ concentrations calculated by GUPIX was to have errors lower than 35\%). Consequently, although generally, $3.0 \mathrm{MeV}$ is often considered the most effective proton energy for PIXE measurements, $3.5 \mathrm{MeV}$ appears to be a good energy for low $\mathrm{Cu}$ concentrations in this kind of samples.

\section{2. $\mathrm{Zn}, \mathrm{Cu}$ and Fe concentration}

Optical images of an adjacent stained section were used to distinguish between areas of lesions and healthy tissue for nuclear microbeam analysis of unstained sections. Fig. 2 shows the region of interest identified on the stained section of tissue with the corresponding $\mathrm{Ca}$ and $\mathrm{Fe}$ PIXE maps of the adjacent unstained section. It is observed that the

Table 1

X-ray production cross sections of $\mathrm{Ca}, \mathrm{Fe}, \mathrm{Cu}$ and $\mathrm{Zn}$ for protons $2.1 \mathrm{MeV}$ and 3.5 $\mathrm{MeV}$ calculated by GUPIX code (see text) and the ratio of the values between both energies.

\begin{tabular}{lccc}
\hline & \multicolumn{2}{c}{ X-ray production cross section (barns) } & Ratio \\
\cline { 2 - 3 } & $2.1 \mathrm{MeV}$ & $3.5 \mathrm{MeV}$ & \\
\hline $\mathrm{Ca}$ & 333.71 & 567.43 & 1.70 \\
$\mathrm{Fe}$ & 93.40 & 217.28 & 2.33 \\
$\mathrm{Cu}$ & 47.36 & 125.51 & 2.65 \\
$\mathrm{Zn}$ & 37.64 & 103.75 & 2.76 \\
\hline
\end{tabular}




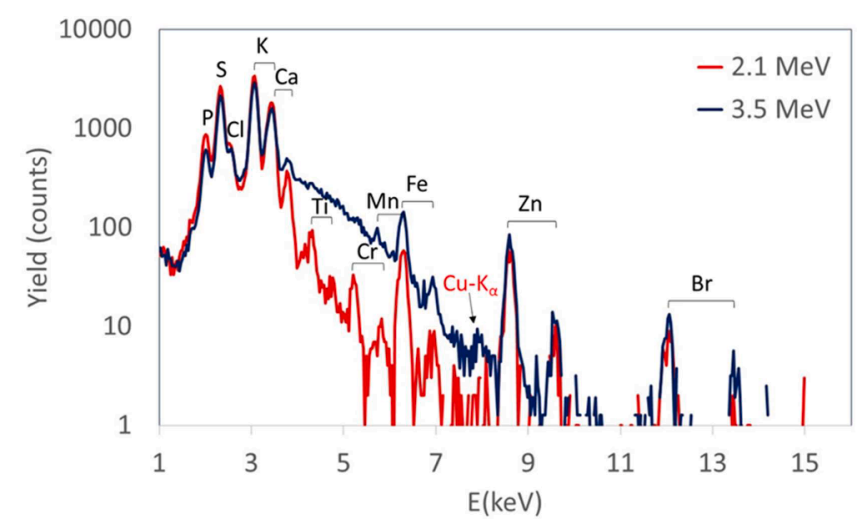

Fig. 1. PIXE spectra of an artery section with $2.1 \mathrm{MeV}$ (red color) and $3.5 \mathrm{MeV}$ (blue color) protons. Both spectra are charged normalized. (For interpretation of the references to color in this figure legend, the reader is referred to the web version of this article.)

structure in lesion part is more chaotic comparing to the smooth muscle wall region.

Fig. 3 shows $\mathrm{Fe}, \mathrm{Cu}$ and $\mathrm{Zn}$ of artery walls without any apparent atherosclerotic lesion of rabbits on normal standard diet and atherosclerotic lesion and non-lesion arterial walls of rabbits on HFD diet (HFD) as well as HFD with Zin supplement. For all groups, these concentrations correspond to the average content enclosed in microscopic areas with a size of about $120 \mu \mathrm{m} \times 120 \mu \mathrm{m}$ included in aorta archsegments close to the heart.

The Fe levels in lesions of animals with both Zinc-rich and HFD diet are significantly higher than that in the non-lesion arteries of animals with a normal standard diet (Fig. 3) in the arch-segment close to the heart. In animals fed a diet without Zinc supplement, the Fe level in lesion was significantly higher than that in the lesion of animals fed a Zinc-rich diet, indicating a positive effect of Zinc supplement in inhibiting the increase of $\mathrm{Fe}$ in this tissues.

The $\mathrm{Cu}$ graph (Fig. 3) shows similar concentrations in non-lesions artery walls for the three studied groups. The $\mathrm{Cu}$ concentrations are significantly lower in atherosclerotic lesions than in non-lesion arteries for the animals fed with HFD without and with Zn supplements. The rabbits fed a diet with Zinc supplements show higher $\mathrm{Cu}$ levels in lesions than those fed only with HFD.
The Zn levels in lesions of animals with both Zinc-rich diet and HFD are significantly lower than that in non-lesion artery walls (Fig. 3). This result agrees with the previous study where areas of about $1 \mathrm{~mm} \times 1 \mathrm{~mm}$ where analysed and sustains the protective role against atherosclerotic lesion formation of this element. In animals fed a diet without Zinc supplement, the $\mathrm{Zn}$ level in lesions was significantly lower than that in the lesion of animals fed a Zinc-rich diet, indicating the positive role of the Zinc supplement in inhibiting the increase of tissue Fe.

These results confirm the observed trend for $\mathrm{Fe}$ and $\mathrm{Zn}$ from our previous work using $2.1 \mathrm{MeV}$, although the values are higher at $3.5 \mathrm{MeV}$. The explanation of this discrepancy could be found in the size of the scanned areas. The former measurements were carried out in areas of at least $1 \mathrm{~mm} \times 1 \mathrm{~mm}$ depending on the growth of the lesion, whereas the selected areas for $3.5 \mathrm{MeV}$ were all of about $120 \mu \mathrm{m} \times 120 \mu \mathrm{m}$. These microscopic areas enable the selection of more accurate regions of interest corresponding to lesions and arterial walls. In addition, in the large scans the contribution from the different layers may affect the elemental concentrations could be different to those obtained for smaller scans. The $\mathrm{Cu}$ levels were successfully measured at $3.5 \mathrm{MeV}$ and follow a similar trend as $\mathrm{Zn}$.

In order to inspect the elemental concentration variations as a function of the artery region, samples from the middle part of the aorta arch were also measured. Fig. 4 compares this region from rabbits on a normal standard diet and the atherosclerotic lesioned and non-lesion arterial walls of rabbits on HFD and with Zinc supplement diet in the levels of $\mathrm{Fe}, \mathrm{Cu}$ and $\mathrm{Zn}$. These bar graphs clearly show that the elevation of Fe in lesions of rabbits on HFD and Zinc-rich diet, and the decrease of Zn level in lesions of rabbits fed with HFD with and without zinc supplement, although a smaller decrease is observed in animals on a Zincrich diet. Whereas the Fe in non-lesion arterial wall of rabbits on HFD with and without zinc rich has not changed much, $\mathrm{Zn}$ levels have been affected and an obvious change is observed. The Cu concentrations seen to decrease in atherosclerotic tissue but its variability is very high and its decline is not as severe as $\mathrm{Zn}$.

Similar elemental behaviours have been noticed in the arch segments close to the heard and in the middle part of the aorta mainly for Fe and $\mathrm{Zn}$. For $\mathrm{Cu}$, its variability in the mid-segment is high and its decrease in lesion areas is not so evident in this segment.

$\mathrm{Fe}, \mathrm{Zn}$ and $\mathrm{Cu}$ are all transition metals, but they demonstrate varied characteristics. Fe is generally considered as a pro-oxidant for its ability of generating free radicals via the Fenton reaction. This fact could

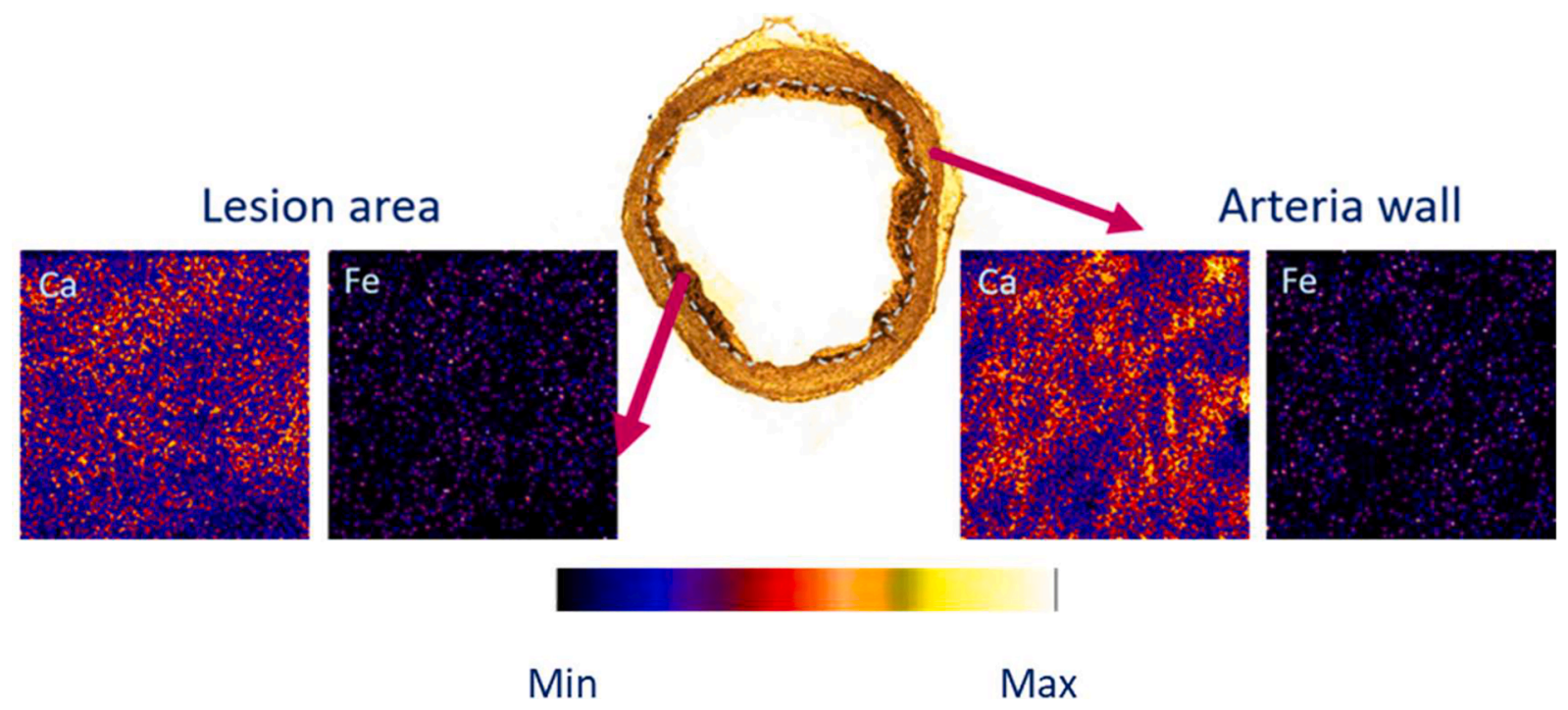

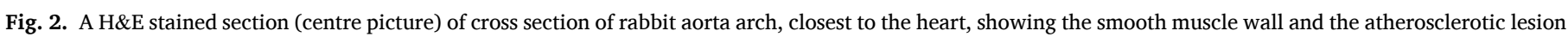

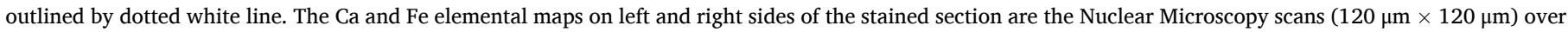
the atherosclerotic lesion area and smooth muscle wall area respectively. 

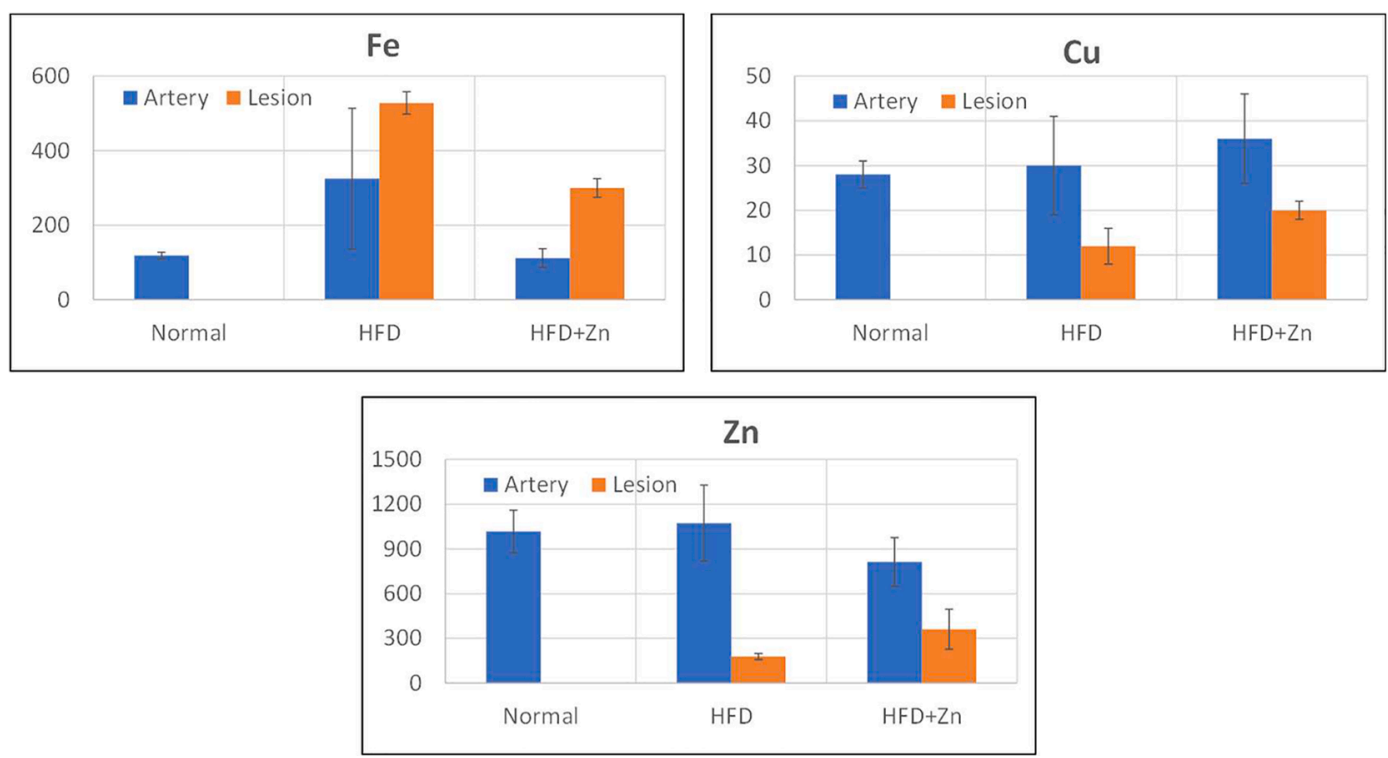

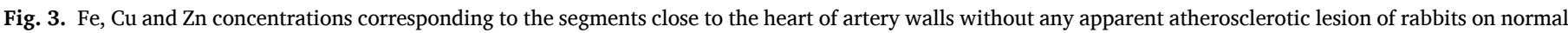

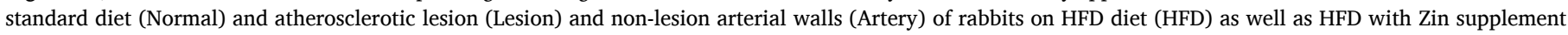
$($ HFD $+\mathrm{Zn})$. The error bars correspond to the standard deviation.
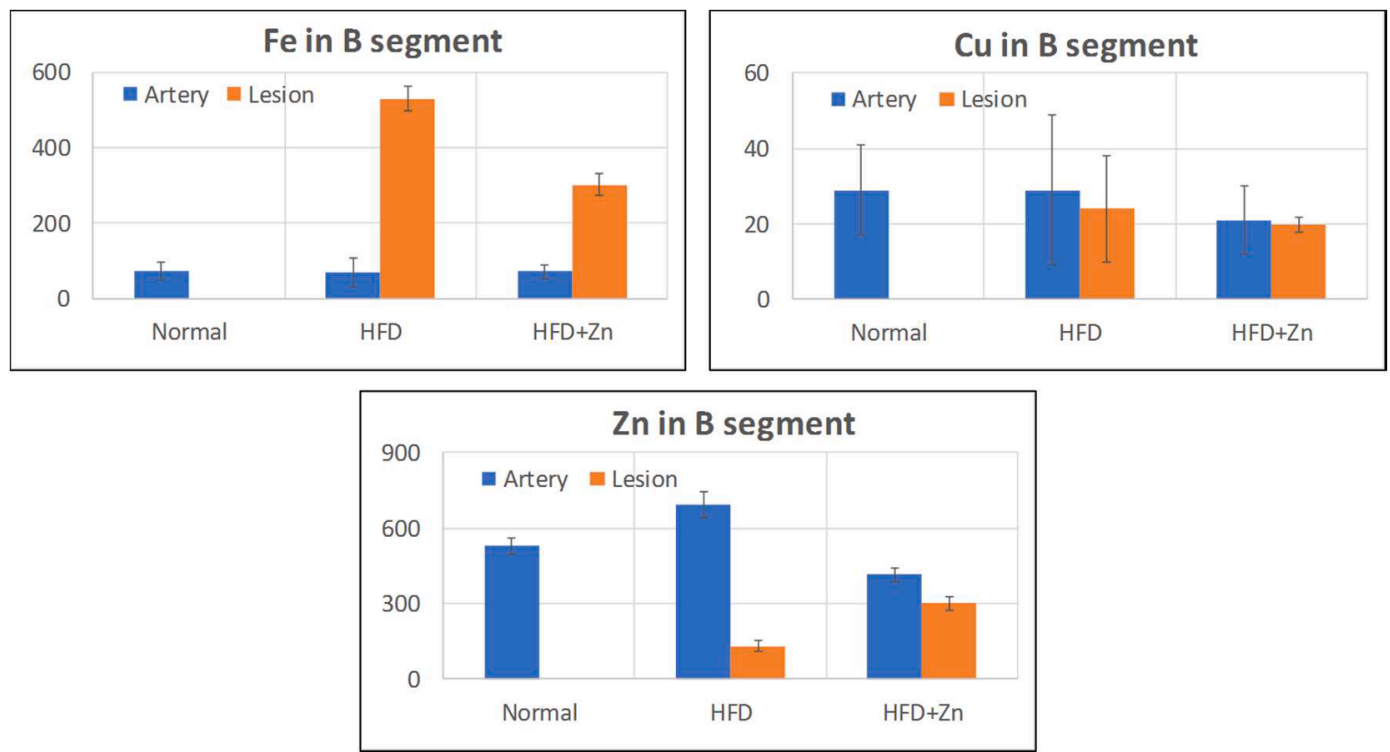

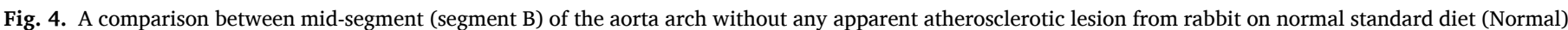

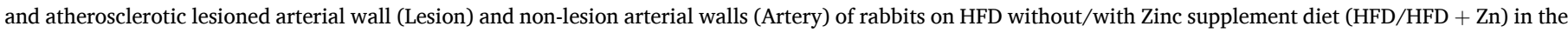
levels of $\mathrm{Fe}, \mathrm{Cu}$ and $\mathrm{Zn}$. The error bars correspond to the standard deviation.

explain the elevated Fe values at lesions regardless of the diet Fig. 3 and Fig. 4. Zn is regarded as an antioxidant because it mostly acts as a Lewis acid. It is essential for normal cell metabolism. This is in accordance with the highest $\mathrm{Zn}$ concentration in the healthy tissue (Fig. 3 and Fig. 4). Cu is present in enzymes in most cells, catalysing biochemical reactions, for example, cytochrome oxidase is involved in energy production and superoxide dismutase protects cells from oxidative damage thus $\mathrm{Cu}$ can be considered as both pro and antioxidant [17]. The greater $\mathrm{Cu}$ presence in the healthy arterial tissue supports its antioxidant role in the artery. Our results show a significant correlation between $\mathrm{Cu}$ and $\mathrm{Zn}$ quantities ( $\mathrm{p}<$ 0.05 ) both in the lesion and the non-lesion artery wall. Atherosclerotic tissue may contain compounds that carry both $\mathrm{Zn}$ and $\mathrm{Cu}$, such as copper zinc superoxide dismutase (CuZnSOD). SODs are enzymes with medical relevance as potential therapeutic agents in diseases related to oxidative stress and are thought to play a major role in moderating the ageing processes. Copper zinc superoxide dismutase (CuZnSOD) catalyses the dismutation reaction of the toxic superoxide radical to molecular oxygen and hydrogen peroxide and thus forms a crucial part of the cellular antioxidant defence mechanism [18].

\section{Conclusions}

The present work shows a specific example of the importance of ion energy in PIXE for the determination of low concentration elements. In particular, low $\mathrm{Cu}$ concentration in artery samples, that previously could not be detected with $2.1 \mathrm{MeV}$, proton beam was determined with 3.5 $\mathrm{MeV}$ proton beam.

The results show beyond doubt that the $\mathrm{Cu}$ level in atherosclerotic 
lesion areas of animal models from both groups with HFD and Zinc-rich diet are lower than that in the arterial wall areas as well as the artery of the normal animal models.

Both Fe and Zn behaviours determined with $3.5 \mathrm{MeV}$ and $2.1 \mathrm{MeV}$ protons are similar, however, differences in the concentration levels have been noticed. These differences could be consequence of the sizes of the scan areas, which were smaller for $3.5 \mathrm{MeV}$ protons. The elemental concentration levels of the large scans may be biased due to the contributions from different layers and they may not match those obtained for smaller areas. The Fe levels in lesions of both groups with HFD and Zinc-rich diet are significantly higher than that in the group with a normal standard diet, and in animals fed a diet without Zinc supplement, the Fe level in lesion was significantly higher than that in the lesion of animals with a Zinc-rich diet. $\mathrm{Zn}$ levels in lesions and nonlesion artery walls of rabbit arteries with a Zinc-rich diet are higher than those with HFD.

Our results show that $\mathrm{Cu}$ and $\mathrm{Zn}$ follow a similar trend and they are believed to have protective role against atherosclerotic lesion formation, therefore, the Cu could play an antioxidant role in protecting the arterial tissue from lesion formation.

\section{CRediT authorship contribution statement}

M.D. Ynsa: . : Investigation, Writing - original draft, Project administration, Writing - review \& editing. J. Ripoll-Sau: Investigation. : . T. Osipowicz: Supervision, Writing - review \& editing. M.B.H. Breese: Supervision, Writing - review \& editing. Minqin Ren: Investigation, Writing - review \& editing.

\section{Declaration of Competing Interest}

The authors declare that they have no known competing financial interests or personal relationships that could have appeared to influence the work reported in this paper.

\section{Acknowledgement}

We thank the technical staff of CMAM for operating the tandem ionbeam accelerator. This work has been partially funded by a UAM-Banco de Santander Interuniversity Cooperation with Asia Grant (2017-2018) and by the Ministerio de Ciencia, Innovación y Universidades (CTQ2017-84309-C2-2-R).

\section{References}

[1] C. Smith, M. J. Mitchinson, O. I. Aruoma, B. Halliwell, Stimulation of lipid peroxidation and hydroxyl-radical generation by the contents of human atherosclerotic lesions, Biochem. J. 286 (1992) 901 - 905, doi: 10.1042/ bj2860901.

[2] K. Jomova, M. Valko, Advances in metal-induced oxidative stress and human disease, Toxicology 283 (2-3) (2011) 65-87, https://doi.org/10.1016/j. tox.2011.03.001.

[3] M. Ren, R. Rajendran, P. Ning, B. Tan Kwong Huat, O. Choon Nam, F. Watt, A. Jenner, B. Halliwell, Zinc supplementation decreases the development of atherosclerosis in rabbits, Free Radical Biol. Med. 41 (2) (2006) 222-225, https:// doi.org/10.1016/j.freeradbiomed.2006.03.017.

[4] B.J. Van Lenten, S.Y. Hama, F.C. de Beer, D.M. Stafforini, T.M. McIntyre, S. M. Prescott, B.N. La Du, A.M. Fogelman, M. Navab, Anti-inflammatory HDL becomes pro-inflammatory during the acute phase response. Loss of protective effect of HDL against LDL oxidation in aortic wall cell cocultures. J. Clin. Invest. 96 (6) (1995) 2758-2767, https://doi.org/10.1172/JCI118345.

[5] L M Klevay, Cardiovascular disease from copper deficiency-a history, J. Nutr. 130 (2000) 489S-492S, doi: 10.1093/jn/130.2.489S.

[6] N. Stadler, R.A. Lindner, M.J. Davies, Direct detection and quantification of transition metal ions in human atherosclerotic plaques: evidence for the presence of elevated levels of iron and copper, Arterioscler. Thromb. Vasc. Biol. 24 (5) (2004) 949-954, https://doi.org/10.1161/01.ATV.0000124892.90999.cb.

[7] B. Bagheri, N. Akbari, S. Tabiban, V. Habibi, V. Mokhberi, Serum level of copper in patients with coronary artery disease, Niger Med. J. 56 (1) (2015) 39, https://doi. org/10.4103/0300-1652.149169.

[8] F. Folkmann, Ion Beam Surface Layer Analysis Vol 2, Meyer et al, New York Plenum, 1976.

[9] M.D. Ynsa, M.A. Ramos, N. Skukan, V. Torres-Costa, M. Jakšić, Highly-focused boron implantation in diamond and imaging using the nuclear reaction ${ }^{11} \mathrm{~B}(\mathrm{p}$, $\alpha)^{8}$ Be, Nucl. Instrum. Methods Phys. Res., Sect. B 348 (2015) 174-177, https://doi. org/10.1016/j.nimb.2014.11.036.

[10] A. Redondo-Cubero, M.J.G. Borge, N. Gordillo, P.C. Gutiérrez, J. Olivares, R. Pérez Casero, M.D. Ynsa, Current status and future developments of the ion beam facility at the centre of micro-analysis of materials in Madrid, Eur. Phys. J. Plus 136 (2) (2021), https://doi.org/10.1140/epjp/s13360-021-01085-9.

[11] M.Q. Ren, F. Watt, K.W. Tan, B. Halliwell, Correlation of iron and zinc levels with lesion depth in newly formed atherosclerotic lesions, Free Radical Biol. Med. 34 (2003) 746-752, https://doi.org/10.1016/s0891-5849(02)01427-2.

[12] P.M. O'Brien, G. Moloney, A. O'Connor, G.J.F. Legge, A versatile system for the rapid collection, handling and graphics analysis of multidimensional data, Nucl. Instr. Meth. B 77 (1993) 52-55, https://doi.org/10.1016/0168-583X(93)95523-8.

[13] J.L. Campbell, GUPIXWIN, available at http://pixe.physics.uoguelph.ca/GUPIX/ main/ (2017).

[14] J.L. Campbell, N.I. Boyd, N. Grassi, P. Bonnick, J.A. Maxwell, The Guelph PIXE software package IV, Nucl. Instrum. Methods Phys. Res., Sect. B 268 (20) (2010) 3356-3363, https://doi.org/10.1016/j.nimb.2010.07.012.

[15] M. Mayer, SIMNRA, a simulation program for the analysis of NRA, RBS and ERDA, Appl. Accel. Res. Ind. 475 (1999) 541-544, https://doi.org/10.1063/1.59188.

[16] K. Ishii, Continuous X-rays produced in light-ion-atom collisions, Radiat. Phys. Chem. 75 (10) (2006) 1135-1163, https://doi.org/10.1016/j. radphyschem.2006.04.008.

[17] M.C. Linder, M. Hazegh-Azam, Copper biochemistry and molecular biology, Am. J. Clin. Nutr. 63 (1996) 797S-811S, https://doi.org/10.1093/ajcn/63.5.797.

[18] E.M. Alissa, S.M. Bahijri, D.J. Lamb, G.A.A. Ferns, The effects of coadministration of dietary copper and zinc supplements on atherosclerosis, antioxidant enzymes and indices of lipid peroxidation in the cholesterol-fed rabbit, Int. J. Exp. Pathol. 85 (2004) 265-275, https://doi.org/10.1111/j.0959-9673.2004.00392.x. 